\title{
Utilização de Undersampling no Projeto de Sistemas de Canalizadores Digitais
}

\author{
Rodrigo Rosal, Adalbery Castro, Aldebaro Klautau. \\ Universidade Federal do Pará - UFPA \\ Laboratório de Processamento de Sinais - LAPS \\ Rua Augusto Corrêa s/n - Guamá - Belém - PA \\ CEP: 66075-900 Fone: +55 91 3201-7674 \\ Emails: rodrigorosal@msn.com, adalbery@ufpa.br, aldebaro@ufpa.br
}

\begin{abstract}
Resumo - Este trabalho aborda projetos de canalizadores digitais usando a técnica de undersampling, a qual permite a amostragem do sinal de interesse a uma taxa menor que indicada por Nyquist. O undersampling é importante porque permite a utilização de conversores $\mathrm{A} / \mathrm{D}$ e $\mathrm{D} / \mathrm{A}$ mais lentos e a redução do custo de processamento do sinal. A literatura carece de trabalhos que considerem o uso de tal técnica de amostragem em conjunto com o projeto de canalizadores. Este artigo visa mostrar detalhes de implementação de duas das mais utilizadas arquiteturas de canalização, a Digital Down Conversion (DDC) e a Polyphase FFT Filter Bank (PFFB) em conjunto com undersampling.
\end{abstract}

Palavras chave - undersampling, canalizador, digital down conversion (DDC), polyphase FFT filter bank (PFFB).

Abstract - This paper focuses on digital channelizer projects using the undersampling technique, which allows sampling a signal in a rate that is smaller than the Nyquist rate. Undersampling is important because it permits the usage of slower $\mathrm{AD}$ and $\mathrm{DA}$ converters and the signal's processing cost reduction. Literature lacks works on the combined use of this technique within the channelizer project. This paper intends to present two of the most used channelization architectures, namely Digital Down Conversion (DDC) and Polyphase FFT Filter Bank (PFFB), combined with the undersampling technique.

Key words - undersampling, channelizer, digital down conversion (DDC), polyphase FFT filter bank (PFFB).

\section{INTRODUÇÃO}

Canalizadores são peças importantes em sistemas que trabalham com mais de um canal de comunicação. Há diferentes tipos de técnicas de canalização e duas das mais populares serão abordadas neste artigo: Digital Down Conversion (DDC) e Polyphase FFT Filter Bank (PFFB). O primeiro é clássico e é usado em muitos sistemas, enquanto que o segundo tem a importante característica de tornar o custo e a complexidade constantes, independente do número de canais a serem extraídos.

A literatura apresenta comparações de canalizadores para aplicações específicas [5] e de undersampling em separado. A contribuição deste trabalho está em uma avaliação das arquiteturas DDC e PFFB em conjunto com a técnica de undersampling.

O trabalho se concentra em aspectos práticos do design de um canalizador tendo como contexto aplicações em telefonia celular. Dependendo do tipo do sistema, existem diferentes restrições na busca dos parâmetros do canalizador. Em um sistema regenerativo, onde há necessidade de decodificação, a taxa de amostragem no final do canalizador deve ser um múltiplo inteiro da taxa de sinalização, como em [1] e [3].

Outra característica importante é a utilização da técnica de undersampling nos conversores A/D e D/A, que permite o uso de conversores mais lentos e, portanto mais baratos. A redução do número de amostras do sinal também implica na redução do custo computacional do sistema.

$\mathrm{Na}$ Seção 2, é feito um breve histórico da evolução dos canalizadores aplicados à telefonia móvel. $\mathrm{Na}$ Seção 3, é discutida e técnica de undersampling, enquanto que na Seção 4, as arquiteturas de canalizadores são apresentadas. A Seção 5 comenta uma aplicação do uso das arquiteturas citadas ao sistema GSM900.

\section{CANALIZADORES}

Um canalizador é a parte de um sistema de comunicação responsável pela extração dos canais de interesse de um sinal banda larga. Em telecomunicações, pode ser usado em estações rádio base, sistemas repetidores, comunicação via satélite, etc.

Inicialmente, os canalizadores eram completamente analógicos. Com o tempo, foram gradualmente sendo digitalizados à medida que os processadores de sinais e os conversores A/D e D/A se tornaram cada vez mais velozes, assim como, foram se desenvolvendo as técnicas de processamento de sinais 
digitais, propiciando o surgimento de novos conceitos como o de radio definido por software (SDR) [1].

SDR foi originalmente desenvolvido para fins militares, mas vem sendo utilizado com propósitos comerciais por causa da sua habilidade em reduzir o custo e a complexidade dos sistemas atuais que utilizam as técnicas de canalização. Baseia-se na utilização de conversores A/D e D/A de banda larga em uma posição que seja o mais próximo possível das antenas e com o máximo das funcionalidades de rádio implementadas no domínio digital [4].

Nos canalizadores analógicos tradicionais, o custo e complexidade do canalizador crescem linearmente com o número de canais a serem extraídos do sinal recebido, o que pode ser evitado utilizando-se as técnicas de SDR, uma vez que a extração dos canais é feita via software e então estará limitada a capacidade de processamento do sistema digital implementado.

Dependendo do produto final do qual o canalizador fará parte e da aplicação (telefonia móvel, por exemplo), existirão pequenas distinções nos parâmetros do canalizador.

A motivação em [3] é o uso de técnicas de canalização para extrair canais de um sinal banda larga e obter um sinal a uma taxa que seja igual a um valor múltiplo da freqüência de símbolo (baud) na saída do canalizador. $\mathrm{O}$ presente trabalho busca também reduzir a velocidade do conversor $\mathrm{A} / \mathrm{D}$ e, conseqüentemente, reduzir o número de amostras a serem tratadas pelos processadores DSP usando a técnica de undersampling.

\section{UNDERSAMPLING}

A técnica de undersampling consiste em amostrar um sinal a uma determinada taxa de amostragem abaixo da taxa sugerida pelo teorema de Nyquist.

Na prática, a amostragem de um sinal analógico de freqüência fa, a uma taxa $f_{s}$, produz componentes em ( $\left.\mathrm{n} \times f_{s}-\mathrm{fa}\right)$ e em ( $\left.\mathrm{n} \times f_{s}+\mathrm{fa}\right)$, onde $\mathrm{n}$ é um número inteiro. Devido a esse fenômeno de aliasing, o teorema de Nyquist requer que o sinal seja amostrado a uma taxa duas vezes maior que fa, o que seria um meio de prevenir que as imagens criadas pelo processo de amostragem não se superponham na primeira zona de Nyquist. Ou seja, este teorema assume que a largura de banda da informação desejada é igual à banda de Nyquist.

Como complemento ao teorema de Nyquist, Shannon mostra que a frequiência de amostragem mínima também é função da largura de banda do sinal a ser amostrado.

Shannon afirma que um sinal analógico com largura de banda $\mathrm{B}$ deve ser amostrado a uma taxa $f_{s}>2 \mathrm{~B}$ para evitar perda de informação. A largura de banda de um sinal pode variar de $\mathrm{DC}$ até $\mathrm{B}$ ou de $\mathrm{f} 1$ até f2, sendo $\mathrm{B}$ $=\mathrm{f} 2-\mathrm{f} 1$. Para este último caso, pode-se utilizar undersampling $[7,8]$.
O fato de o sinal estar amostrado a uma taxa maior que 2B não significa que não houve sobreposição entre as imagens, e isto deve ser observado. Uma análise mais generalizada sobre este problema está mostrada na Figura 1, onde a mínima taxa de amostragem necessária para evitar sobreposição entre imagens é função da componente de máxima freqüência e da largura de banda do sinal amostrado. As áreas hachuradas indicam as regiões que correspondem a freqüências válidas, ou seja, onde não ocorre sobreposição entre imagens.

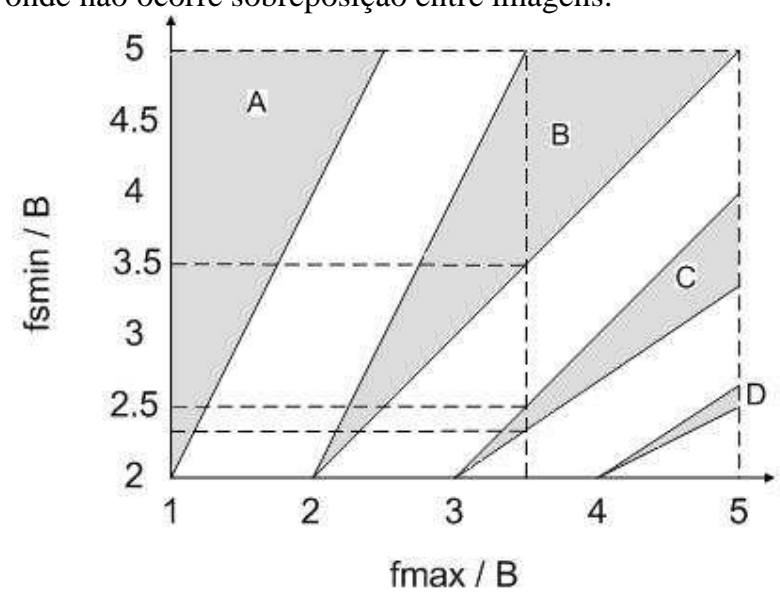

Figura 1: Mínima taxa de amostragem $\left(f_{s}\right)$ como função da máxima componente de freqüência $\left(f_{\max }\right)$ e da largura de banda $(B)$ do sinal amostrado.

Na Figura 1, a primeira área hachurada (A) é a região onde se utiliza o teorema de Nyquist, ou seja, a $f_{s}$ é maior ou igual ao dobro da maior componente do sinal. As demais áreas $(\mathrm{B}, \mathrm{C}, \mathrm{D})$ representam regiões onde se pode amostrar o sinal abaixo da taxa estabelecida por Nyquist, sem que ocorra sobreposição entre imagens.

Em muitas aplicações, esta técnica reduz consideravelmente a demanda requerida pelo conversor A/D. Por exemplo, um sinal centrado em $30 \mathrm{MHz}$ e com uma largura de banda de $10 \mathrm{MHz}$ (Figura 2a) precisaria de um conversor A/D de no mínimo 70 MSPS, seguindose o critério de Nyquist, porém utilizando-se undersampling seria possível amostrar o mesmo sinal com um conversor A/D de 25 MSPS. Esta operação esta mostrada na Figura $2 b$.

$\mathrm{O}$ efeito de aliasing pode ser utilizado em favor do projetista de processamento digital, uma vez que a partir de uma escolha adequada de frequiência de amostragem do conversor $\mathrm{A} / \mathrm{D}$, o projetista pode escolher a posição da banda de interesse e executar uma ação de translado do sinal no domínio das frequiências. Inclusive, sendo possível ocorrer o translado para a banda base, como mostra a Figura $2 b$.

Isto pode ser feito porque as imagens criadas pelo fenômeno de aliasing são cópias idênticas do sinal original, o que significa dizer que o projetista pode escolher qualquer uma delas como sinal de interesse, filtrando as imagens indesejadas e mantendo o cuidado de evitar que as imagens se sobreponham. 

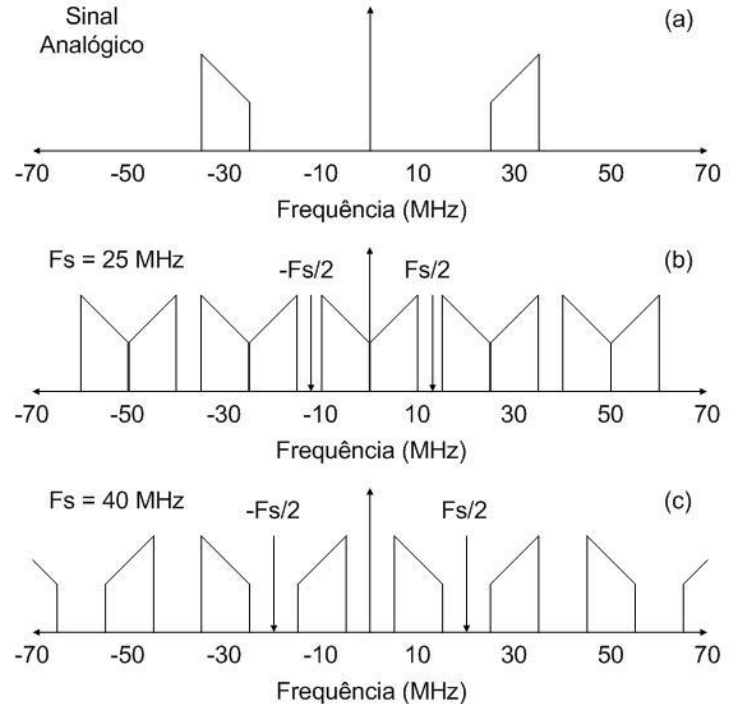

Figura 1: Exemplo da utilização da técnica de undersampling. (a) Sinal analógico a ser amostrado, (b) sinal amostrado a uma taxa de $25 \mathrm{MHz}$ e (c) sinal amostrado a $40 \mathrm{MHz}$.

Outro fator importante diz respeito à inversão espectral, que acontece quando a imagem das freqüências positivas aparece com o formato da imagem das freqüências negativas e a imagem das freqüências negativas aparece no formato das freqüências positivas, Figura 2c.

A inversão espectral ocorre quando a frequiência escolhida para o conversor $\mathrm{A} / \mathrm{D}$, sem provocar superposição de imagens, é maior que a frequiência de centro da banda de interesse e menor que a frequiência de Nyquist. A inversão espectral pode ser facilmente removida via software, fazendo-se uma simples transformação algébrica, que consiste na multiplicação do sinal de entrada real por $(-1)^{\mathrm{n}}$, onde $\mathrm{n}=0,1,2,3$, etc. $\mathrm{O}$ que é equivalente a misturar a seqüência de entrada com uma sequiência senoidal de freqüência $f_{s} / 2$ [8].

No domínio das freqüências, esta transformação equivale girar o lado positivo das freqüências, de 0 à $f_{s} / 2$, com relação a $f_{s} / 4$ e girar o lado negativo das freqüências, de $-f_{s} / 2$ à 0 em torno de $-f_{s} / 4$.

Uma maneira de se evitar a inversão espectral é escolher uma frequiência para o conversor $\mathrm{A} / \mathrm{D}$, sem provocar superposição de imagens, menor que a frequiência de centro da banda de interesse.

Há uma situação onde não é possível realizar undersampling, que é quando a componente de freqüência mais baixa do sinal é menor que a largura de banda do sinal. Neste caso, deve-se amostrar obedecendo ao critério de Nyquist.

\section{ARQUITETURAS DE CANALIZADORES}

As arquiteturas que serão mostradas nesta seção são arquiteturas predominantes nos sistemas de comunicação: a Digital Down Conversion (DDC) e a Polyphase FFT Filter Bank (PFFB).

\section{A. Digital Down Conversion (DDC)}

Um canalizador DDC usa a técnica da heterodina. Nesta técnica, o sinal banda larga é misturado com um par de osciladores locais em quadratura centrados na frequiência que se deseja rebaixar para banda base.

Depois dos misturadores, há um processo de filtragem passa baixas, que tem por finalidade rejeitar imagens criadas no processo de translado na freqüência.

Finalmente, o sinal é decimado para reduzir a freqüência de amostragem a um valor mínimo necessário para suportar o canal. A Figura 3 mostra um canalizador DDC de k canais.

O processo de filtragem pode ser composto por um filtro FIR ou por um filtro de Hogenauer, também chamado de filtro CIC (Cascaded Integrator Comb). Normalmente, o filtro CIC é seguido por filtros FIR de compensação com um número de coeficientes programáveis para aperfeiçoar o processo de filtragem.

Algumas das vantagens desta arquitetura são: flexibilidade na seleção, tanto da freqüência de centro dos canais, quanto da largura de banda; facilidade para adicionar e remover canais; design independente dos canais dentro do canalizador.

Como desvantagem, pode-se citar que o custo e a complexidade da arquitetura variam linearmente com a quantidade de canais a serem extraídos.

No design de um canalizador utilizando a arquitetura DDC e a técnica de undersampling, a primeira ação a ser tomada é encontrar as bandas de frequiência para o conversor $\mathrm{A} / \mathrm{D}$ nas quais não haverá superposição entre as imagens criadas. Para tanto, precisa-se conhecer a largura de banda do sinal de interesse e a porção do espectro na qual este sinal está localizado.

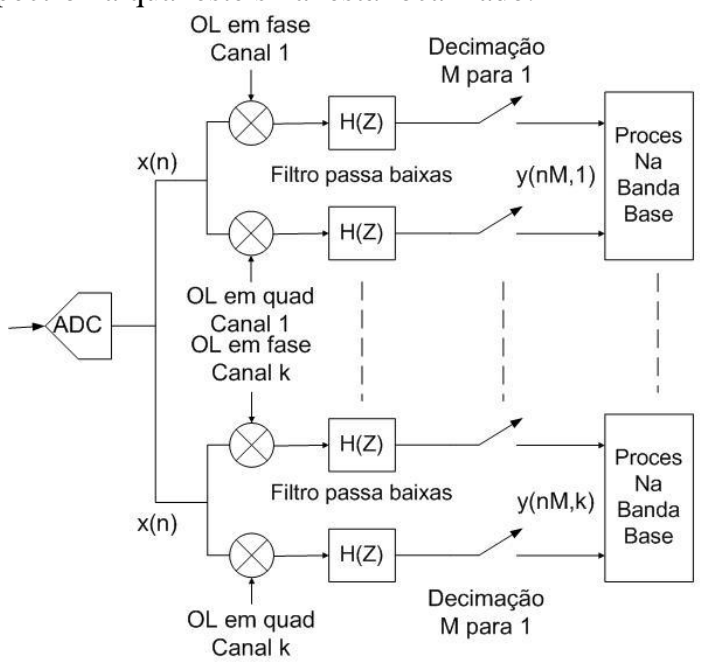

Figure 3: Arquitetura DDC de k canais.

Em sistemas regenerativos, a freqüência de amostragem do sinal decimado $f_{\text {dec }}$, que molda o sinal $\mathrm{y}(\mathrm{nM}, \mathrm{k})$ deve ser um múltiplo inteiro da taxa de sinalização [3]. Então,

$$
f_{\text {dec }}=H \times f_{b}
$$


onde $H$ é o número de amostras por símbolo e $f_{b}$ é a taxa de sinalização.

A partir da Eq. (1) e do fator de decimação $M$, a ser utilizado, pode-se encontrar a freqüência do conversor A/D $\left(f_{s}\right)$.

$$
f_{s}=M \times f_{d e c}=M \times H \times f_{b}
$$

Lembrando que o valor de $f_{s}$, calculado usando a Eq. (2), deve estar dentro de uma das bandas de frequiência encontradas para a utilização da técnica de undersampling sem haver sobreposição de imagens. E o valor de $f_{d e c}$ deve satisfazer o critério de Nyquist.

Uma forma alternativa de efetuar o projeto de um canalizador utilizando a arquitetura DDC é ignorar a restrição de que a freqüência $f_{d e c}$ deve ser um múltiplo inteiro da taxa de sinalização, projetar o canalizador como se ele fosse não regenerativo, e realizar uma operação de reamostragem no final do processo, para que a freqüência do sistema após a reamostragem atenda a restrição anteriormente ignorada. Porém, o fato de o processo de reamostragem ser muito custoso pode inviabilizar a utilização deste método [3].

\section{B. Polyphase FFT Filter Bank (PFFB)}

A arquitetura PFFB, mostrada na Figura 4, é composta de um comutador que entrega amostras consecutivas aos $M$ filtros, executando uma operação de decimação, uma vez que cada filtro recebe uma amostra a cada $M$ amostras. Esta operação de decimação rebaixa todos os $M$ canais centrados em freqüências que são múltiplas da freqüência de amostragem de saída para a banda base.

Uma observação importante é que todos os canais deverão estar centrados em freqüências que sejam múltiplos inteiros da freqüência de amostragem de saída, para que a operação de decimação rebaixe corretamente os canais, utilizando a técnica de undersampling. O que significa dizer que os canais deverão estar igualmente espaçados no domínio das frequiências.

Os termos em cada processo de filtragem têm perfis de fase únicos por causa das suas diferentes freqüências de centro e devido à diferença de tempo com a qual foram entregues na entrada dos seus respectivos filtros. Cada freqüência de centro sofre uma mudança de fase que depende do atraso na entrada do seu processo de filtragem.

O filtro é chamado polifásico, por causa do modo como é particionado. Cada um dos $M$ filtros, com coeficientes $\mathrm{h}(\mathrm{r}+\mathrm{n} M)$, é formado com um atraso $\mathrm{r}$ e é incrementado a cada $\mathrm{M}$ coeficientes. Como os atrasos iniciais são únicos para cada caminho, eles produzem perfis diferentes de fase linear. Então, devido aos diferentes perfis de fase, o filtro é chamado de polifásico.

A FFT (Fast Fourier Transform) realiza uma soma coerente das fases, extraindo do conjunto de freqüências de centro, a freqüência que corresponde a cada perfil de fase.

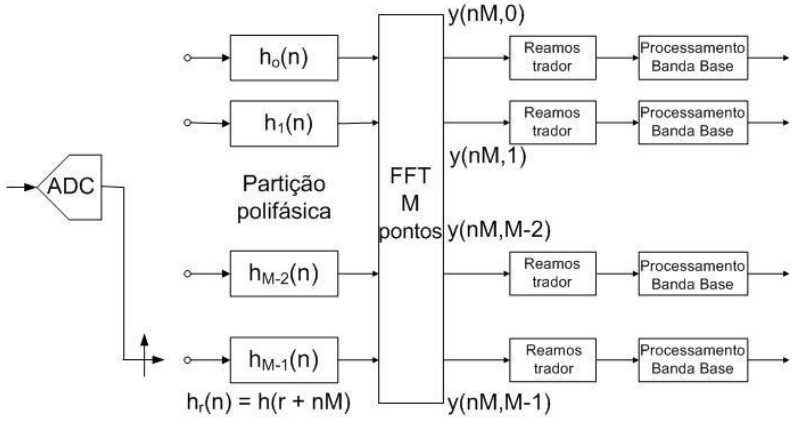

Figura 4: Arquitetura PFFB de M canais.

Em geral, o número de polifases deve ser igual ao fator de decimação, quando isso acontece $o$ canalizador é dito como estando maximamente decimado.

Uma vantagem desta arquitetura é o baixo custo computacional para uma grande quantidade de canais, requerendo um único filtro FIR e a FFT para separar todos os canais, com a FFT normalmente conduzindo a complexidade do canalizador.

Entre as desvantagens pode-se citar que os canais devem estar igualmente espaçados; que a adição ou remoção de canais não é prática; e que os canais não podem ser projetados independentemente.

No design de um canalizador usando a arquitetura PFFB e a técnica de undersampling, a primeira ação a ser tomada, como no caso da arquitetura DDC, é encontrar as bandas de frequiência para o conversor $\mathrm{A} / \mathrm{D}$ nas quais não haverá superposição entre as imagens criadas. Para tanto, precisa-se conhecer a largura de banda do sinal de interesse e a porção do espectro na qual este sinal está localizado.

Em sistemas regenerativos, a frequiência de amostragem $f_{\text {dec }}$, que molda o sinal $\mathrm{y}(\mathrm{nM}, \mathbf{M})$ deve ser um múltiplo inteiro da taxa de sinalização [3] e também deve ser um múltiplo inteiro da largura de banda do canal, $B_{c h}$, então,

$$
f_{d e c}=\text { Mínimo Múltiplo } \operatorname{Comum}\left(f_{b}, B_{c h}\right)
$$

Como a taxa de sinalização e a largura de banda do canal são normalmente dois números de ordem elevada, e o custo computacional da arquitetura PFFB depende basicamente da FFT. É comum deixar a freqüência de amostragem $f_{\text {dec }}$ depender somente da largura de banda do canal, e depois do processo de canalização, reamostrar o sinal y(nM, M) a uma taxa que seja um múltiplo inteiro da taxa de sinalização [3]. Então,

$$
f_{\text {dec }}=B_{c h}
$$

para um canalizador maximamente decimado.

A partir da Eq. (4) e do fator de decimação $M$, pode-se encontrar a freqüência do conversor A/D.

$$
f_{s}=M \times f_{d e c}=M \times B_{c h}
$$

Relembrando que o valor de $f_{s}$, calculado usando a Eq. (5), deve estar dentro de uma das bandas de 
freqüência encontradas para a utilização da técnica de undersampling sem haver sobreposição de imagens.

Pode-se tornar arbitrária a taxa de amostragem na saída do canalizador, ou seja, a $f_{d e c}$ não precisaria ser igual à largura de banda do canal. $\mathrm{O}$ que equivaleria a dizer que o sinal pode ser decimado uma taxa arbitrária [2].

\section{SIMULAÇÕES}

Para as simulações, foram considerados os padrões do sistema GSM900, com os canais com largura de banda de $200 \mathrm{kHz}$ e rebaixados para uma frequiência intermediária, estando o primeiro canal centrado em 57,8 $\mathrm{MHz}$, o último em 82,4 MHz e espaçados de 0,2 MHz.

Foram considerados ainda a frequiência de bits $f_{b}$ $(270 \mathrm{KHz})$, a largura de banda total $(25 \mathrm{MHz})$ e o fato de canais adjacentes não serem utilizados.

A simulação foi realizada gerando-se seqüências aleatórias de bits para cada canal utilizado, que serão codificadas utilizando a modulação GMSK (Gaussian Minimum Shift Keying). As sequiências foram então somadas e adicionadas a ruído aditivo gaussiano branco.

Foi simulado o efeito de undersampling causado pelo conversor $\mathrm{A} / \mathrm{D}$, para então ser realizado processo de canalização do sinal para separação dos canais. Então, foi realizada a decodificação de cada canal, para obter a taxa de erro de bit. A Figura 5 mostra o diagrama de blocos da simulação.

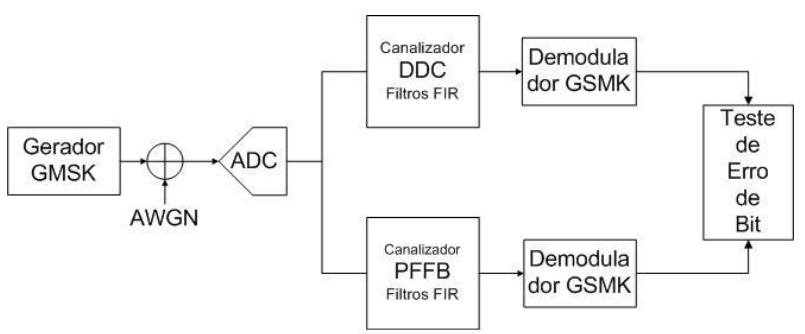

Figura 5: Diagrama de blocos da simulação.

A primeira ação a ser tomada na elaboração do projeto de um canalizador utilizando a técnica de undersampling é o cálculo das freqüências nas quais não haverá sobreposição de imagens. Para isso, é necessário conhecer os valores da largura de banda do sinal (25 $\mathrm{MHz}$ ), da freqüência de centro da banda de todo o sinal $(70,1 \mathrm{MHz})$ e da componente de máxima frequiência $(82,6$ $\mathrm{MHz}$ ). Então, com o auxílio da Figura 1, é possível encontrar dois intervalos de taxa de amostragem $\mathrm{f}_{\mathrm{s}}$ onde não ocorrerá sobreposição das imagens usando undersampling.

- Intervalo 1: 55,0667 MHz $<f_{s}<57,6 \mathrm{MHz}$

- Intervalo 2: $82,6 \mathrm{MHz}<f_{s}<115,2 \mathrm{MHz}$

- Intervalo 3: $f_{s}>165,2 \mathrm{MHz}$

No intervalo 1, utiliza-se undersampling sem inversão do espectro, enquanto que no intervalo 2 , há inversão espectral. O terceiro intervalo possível, que não é mostrado na Figura 1, refere-se à amostragem sem undersampling, ou seja, respeitando o teorema de Nyquist.

\section{A. Projeto DDC}

Primeiramente, escolhe-se um valor inteiro positivo para determinar o número de amostras por símbolo, e então, calcula-se a freqüência de amostragem de saída do canalizador $f_{d e c}$. O fator inteiro escolhido nas simulações foi $H$ igual a quatro, vide Eq. (1). Logo, a frequiência de saída do sistema foi $1080 \mathrm{kHz}$.

A taxa de amostragem do conversor $\mathrm{A} / \mathrm{D}$, o fator de decimação e os valores das freqüências de centro após o undersampling para cada intervalo estão mostrados na Tabela I.

Nota-se, claramente, a capacidade do efeito de undersampling em reduzir a velocidade necessária para os conversores $\mathrm{A} / \mathrm{D}$ e $\mathrm{D} / \mathrm{A}$.

Tabela I: Taxa de amostragem do conversor A/D, fator de decimação e freqüências de centro após undersampling

\begin{tabular}{|c|c|c|c|}
\hline & Intervalo 1 & Intervalo 2 & Intervalo 3 \\
\hline $\begin{array}{c}\text { Fator de } \\
\text { decimação }\end{array}$ & 52 & 90 & 160 \\
\hline $\begin{array}{c}\text { Freqüência de } \\
\text { amostragem do } \\
\text { conversor A/D }\end{array}$ & $56160 \mathrm{kHz}$ & $97200 \mathrm{kHz}$ & $172800 \mathrm{kHz}$ \\
\hline $\begin{array}{c}\text { Freqüências de } \\
\text { centro após }\end{array}$ & $1640 \mathrm{kHz}:$ & $39400 \mathrm{kHz}:$ & $57800 \mathrm{kHz}:$ \\
undersampling & $26240 \mathrm{kHz}:$ & $200 \mathrm{kHz}:$ & $200 \mathrm{kHz}:$ \\
\hline $\begin{array}{c}\text { Freqüências de } \\
\text { centro após } \\
\text { correção do } \\
\text { espectro }\end{array}$ & Não se aplica & $\begin{array}{c}14800 \mathrm{kHz} \\
8200 \mathrm{kHz}:\end{array}$ \\
\hline
\end{tabular}

As freqüências de centro após undersampling podem ser calculadas a partir da convolução da seqüência de pulsos devido à amostragem com as frequiências de centro do canal. Enquanto que, o valor após a correção espectral é obtido a partir da convolução das freqüências de centro após undersampling $\operatorname{com} f_{s} / 2$.

A taxa de erro de bit por SNR encontrada para decodificação de um canal no intervalo 1 (menor $\mathrm{f}_{\mathrm{s}}$ ) está listada na Tabela II.

Tabela II: Taxa de erro de bit por SNR.
\begin{tabular}{|c|c|c|c|c|c|}
\hline SNR & -2 & -1 & 0 & 1 & 2 \\
\hline BER & 0.020288 & 0.019052 & 0.01802 & 0.017272 & 0.016776 \\
\hline
\end{tabular}

\section{B. Projeto PFFB}

Escolheu-se a freqüência de amostragem de saída do canalizador como sendo a largura de banda de um canal. Então, a freqüência de saída do sistema foi 200 $\mathrm{kHz}$.

A taxa de amostragem do conversor $\mathrm{A} / \mathrm{D}$, o fator de decimação, o número de pontos da FFT e os valores das freqüências de centro após o undersampling para cada intervalo estão mostrados na Tabela III.

O cálculo das frequiências de centro após undersampling e após correção do espectro é feito da mesma forma citada no projeto DDC. 
Tabela III: Taxa de amostragem do conversor A/D, fator de decimação, número de pontos da FFT e freqüências de centro após undersampling.

\begin{tabular}{|c|c|c|c|}
\hline & Intervalo 1 & Intervalo 2 & Intervalo 3 \\
\hline $\begin{array}{c}\text { Fator de } \\
\text { decimação }\end{array}$ & 280 & 486 & 864 \\
\hline $\begin{array}{c}\text { Freqüência de } \\
\text { amostragem do } \\
\text { conversor A/D }\end{array}$ & $56000 \mathrm{kHz}$ & $97200 \mathrm{kHz}$ & $168000 \mathrm{kHz}$ \\
\hline $\begin{array}{c}\text { Número de pontos } \\
\text { da FFT }\end{array}$ & 280 & 486 & 864 \\
\hline $\begin{array}{c}\text { Freqüências de } \\
\text { centro após } \\
\text { undersampling }\end{array}$ & $\begin{array}{c}1800 \mathrm{kHz}: \\
200 \mathrm{kHz}:\end{array}$ & $\begin{array}{c}39400 \mathrm{kHz}: \\
200 \mathrm{kHz}:\end{array}$ & $\begin{array}{c}57800 \mathrm{kHz}: \\
14800 \mathrm{kHz}:\end{array}$ \\
\hline $\begin{array}{c}\text { Freqüências de } \\
\text { centro após } \\
\text { correção do } \\
\text { espectro }\end{array}$ & Não se aplica & $\begin{array}{c}9200 \mathrm{kHHz}: \\
200 \mathrm{kHz}:\end{array}$ & Não se aplica \\
\hline
\end{tabular}

\section{CONCLUSÃO}

A técnica de undersampling permite uma redução considerável da freqüência de amostragem requerida para o conversor $\mathrm{A} / \mathrm{D}$, e, conseqüentemente, a redução do custo computacional requerido pelo sistema, quando comparados com as taxas de amostragem indicadas pelo teorema de Nyquist. É possível, mesmo com menos amostras, utilizar undersampling em projetos de canalizadores DDC e PFFB sem comprometer a decodificação do sinal.

\section{AGRADECIMENTOS}

Esse trabalho foi desenvolvido com apoio da empresa Brasilsat Harald S.A. e da CAPES.

\section{REFERÊNCIAS}

[1] F. J. Harris, C. Dick e M. Rice, "Digital Receivers and Transmitters Using Polyphase Filter Banks for Wireless Communications", IEEE Transactions on Microwave Theory and Techniques, Abril 2003.

[2] F. J. Harris e C. Dick, "Performing Simultaneous Arbitrary Spectral Translation and Sample Rate Change, in Polyphase Interpolating or Decimating Filters in Transmitters and Receivers".

[3] K. C. Zangi e R. D. Koilpillai, "Software Radio Issues in Cellular Base Stations", IEEE Journal on Selected Areas in Communications, Abril 1999.

[4] K. C. Zangi e R. D. Koilpillai, "Efficient Filter Bank Channelizers for Software Radio Receivers", IEEE, 1998.

[5] L. Pucker, "Channelization Techniques for Software Defined Radio", SDR forum, 2003.

[6] P. P. Vaidyanathan, "Multirate Digital Filters, Filter Banks, Polyphase Networks, and Applications: a Tutorial", IEEE Proceedings, janeiro 1990.

[7] P. McCormack, "Effects and Benefits of Undersampling in High Speed ADC Applications", Design \& Elektronik (Alemanha), maio 2004.

[8] R. G. Lyons, Understanding Digital Signal Processing, Prentice Hall, 1edição, 2001.
[9] S. Haykin, Communication Systems, John Wiley \& Sons, 4 edição, 2001.

[10] H. Harada e R. Prasad, Simulation and Software Radio for Mobile Communications, 2002.

[11]R. G. Vaughan, N. L. Scott e D. R. White,"The Theory of Bandpass Sampling”, IEEE Transactions on Signal Processing, setembro 1991. 